\title{
Integrated management systems in design and survey organizations
}

\author{
Anastasia Aksenova ${ }^{1, *}$ \\ ${ }^{1}$ Moscow State University of Civil Engineering, 26, Yaroslavskoe shosse, Moscow, 129337, Russia
}

\begin{abstract}
The relevance of the research topic is due, primarily, to the development trends of the construction industry of the Russian Federation in the face of declining quality of building products, the main reasons are low level of qualification, the lack of systematic monitoring at all stages of the production cycle, the deterioration of product quality of design and survey organizations. Today, the process of carrying out design and survey works is implemented in the conditions of an outdated regulatory and technical base, incorrectly drawn up technical tasks, and in some cases without prior approval of the technological part. The production cycle of construction products begins with the stage of design and survey work. The defining direction in the process of forming such characteristics of finished construction products as quality, its safety for the environment and consumers is the continuous improvement of this stage in changing conditions. Ensuring effective management of the process of creating design and estimate documentation allows to monitor, control and improve the quality, environmental friendliness and safety of construction products at each stage of its design. High indicators of quality, safety for the environment and consumers of design and survey products depend on the combined interaction of internal factors of the organization that ensure its sustainable development in constantly changing external conditions. It is at the stage of design and survey work that decisions are made on the criteria of economic and environmental efficiency of construction and operation of construction products. Design and survey organizations act as guarantors of both the safety of the future object and the payback of the resources invested by the customer, laying in the products the most cost-effective and modern technical solutions from the point of view of human and environmental safety.
\end{abstract}

\section{Introduction}

Analysis of the design and survey organizations have shown a reduction in the number of highly qualified personnel in the field of design and engineering researches in a context of increasing number of new project and exploration organizations, low professional level, as well as a sharp decline in the number of design and survey organizations, technologically specialized industry features, which leads to a lack of developed technical solutions of scientific and technical progress, economic feasibility, energy - saving technologies.

\footnotetext{
* Corresponding author: AksenovaAA@mgsu.ru
} 
The reasons for the decrease in the efficiency of design and survey organizations established as a result of the study indicate non-compliance with the Shewhart-Deming's PDC(S)a Quality cycle [1-2] focused on continuous improvement, the lack or ineffective use of quality management tools, ecology and safety of work. As a comprehensive solution to the identified problems, a methodology has been developed to ensure the effectiveness of the design and survey organization in the context of an integrated management system. The proposed methodology is based on the implementation of two main stages: I) creation of an effectively functioning integrated management system; II) implementation of an integrated approach to continuously improve the functioning of the integrated management system.

\section{Materials and Methods}

The paper contains the results of an analytical approach based on an assessment of the current state of the construction industry in Russia, particularly of the design and survey organizations.

Also the theoretical and methodological basis of the research is the fundamentals of management and economic theory, the fundamentals of system analysis, the theory of selforganization, the triune concept of sustainable development, the concept of universal quality management, the theory of systems functioning, scientific works and practical experience of domestic and foreign specialists in the field of quality management, work safety and environmental protection.

The data used in this study are taken from the databases of Russian Federal State Statistics Service (Rosstat).

\section{Experimental part}

The result of the first stage of the developed methodology is the organization of work on the:

- creation and implementation of an integrated management system (IMS),

- identification of the functioning of the existing management system of the design and survey organization,

- implementation of measures for the implementation of the developed IMS,

- ensuring and analyzing its functioning.

The implementation of the second stage of the proposed methodology allows, based on the results of the analysis of the IMS functioning for a certain period of time, to develop a set of measures to improve the efficiency, effectiveness and adaptability of its functioning. The implementation of the developed measures is based on the results of the previously calculated expected cumulative effect of the IMS operation and prevents the appearance of financial losses from investments in dubious investment projects.

The main difference between creating an integrated management system and creating autonomous quality management systems, environmental protection and labor safety is the formation of a set of integrative properties. In other words, the target function of the created integrated management system will be [3]:

$$
\sum_{i=1}^{i=n} \sum_{j=1}^{j=N} k_{i j}=K_{0},\left.K_{0} \rightarrow\right|_{\min } ^{\max }
$$

$\mathrm{k}_{\mathrm{ij}}$ - performance indicator for each of the three components of the system (quality management system (QMS), environmental management system (EMS), health and safety management system (HaSMS) i for each element of the system $\mathrm{j}$.

Elements in an integrated management system are actions performed by employees of a design and survey organization, the purposeful combination of which forms the IMS 
processes. Then $\mathrm{N}$ will be equal to the number of final homogeneous processes or their constituent actions determined during the analysis of the design and survey organization.

$\mathrm{K}_{0}$ - the target value of the function under study, which, when creating an integrated management system based on the principle of combining the three components of its systems, should strive to form integrative properties. The set of integrative properties must be optimal, that is, ensure that the maximum or minimum value is reached. When creating an integrated management system, the extremum of the function is to ensure the lowest operating costs under the following conditions:

$$
\mathrm{k}_{\mathrm{i}}=3 ; \mathrm{N} \rightarrow \min
$$

The creation of an integrated management system is based on the implementation of the principle of combination, which implies the superposition of elements of three systems on top of each other, completely eliminating the duplication of homogeneous actions and processes [4]. Since the combination principle is a higher-order heuristic technique used in creating complex systems, the result of combining the elements of the three components of an integrated management system is the appearance of a new property that characterizes the synergistic effect. Integrative properties in the management system are laid down when designing its structure. The description of the integrated management system structure is based on indicators:

- compactness, which determines the value of connections between system elements:

$$
Q=\sum_{i=1}^{n} \sum_{j=1}^{n} d_{i j}, i \neq j
$$

$d_{i j}-$ the minimum value of the relationship between elements $i$ and $j$.

- connectivity, which determines the continuity of the implementation of elements, the presence of discontinuities in the system structure:

$$
0,5 \sum_{\mathrm{i}=1}^{\mathrm{n}} \sum_{\mathrm{j}=1}^{\mathrm{n}} \mathrm{a}_{\mathrm{ij}} \geq \mathrm{n}-1, i \neq j
$$

$\mathrm{a}_{\mathrm{ij}}$ - elements of an integrated management system, $\mathrm{n}$ is the number of vertexes in the structural model of the system.

redundancy, which determines the optimal value of connections in the system:

$$
\mathrm{R}=0,5\left[\sum_{\mathrm{i}=1}^{\mathrm{n}} \sum_{\mathrm{j}=1}^{\mathrm{n}} \mathrm{a}_{\mathrm{ij}}\right] \frac{1}{\mathrm{n}-1}-1
$$

in case $\mathrm{R}>0$ - the integrated management system is characterized by maximum redundancy of connections,

in case $\mathrm{R}=0$ - the integrated management system is characterized by minimal redundancy of links,

in case $\mathrm{R}<0$ - characterizes the structure of an integrated management system as incoherent.

The System, which has $\mathrm{R}>0$, is the most resistant to external factors. At the stage of creating an integrated management system, the main purpose of the studied relationships will be to provide processes with resources. At the initial stage of creating an integrated management system (IMS), it is necessary to determine the type of its future structure, which will be fundamental in calculating the indicators of compactness, connectivity and redundancy. 

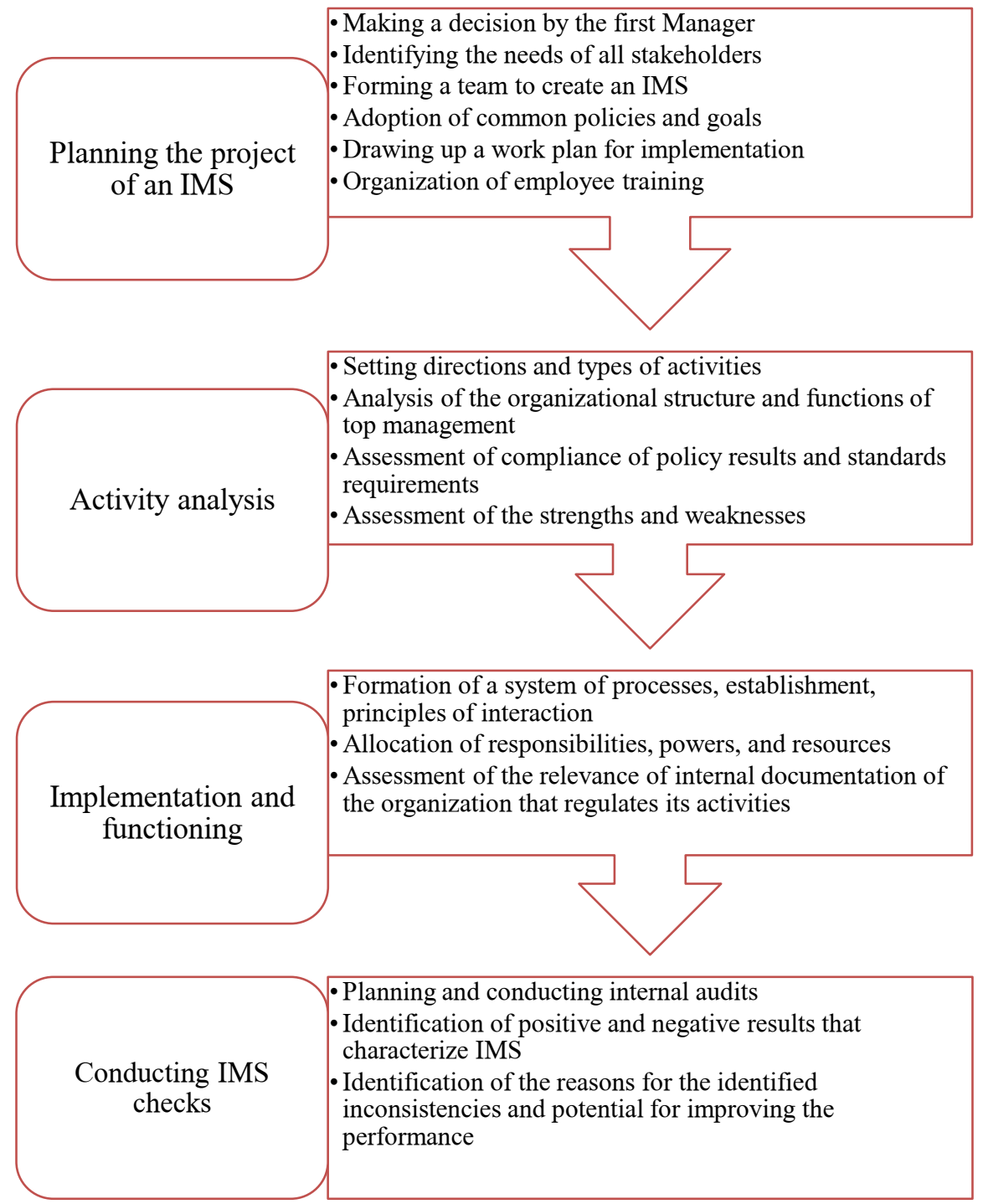

Fig. 1. Block diagram of the algorithm for creating an IMS in a design and survey organization

In the study we consider the creation of an IMS in a design and survey organization as a parallel implementation and integration of three systems. Since the requirements of the ISO 14001 and OHSAS 18001 standards are similar in many ways and are easily applied to the organization's activities in the aggregate, in contrast to the requirements of ISO 9001, the created system will have a tree structure. During the construction, we will introduce the concepts of "general" and "special" measures for the implementation of IMS, which will meet the requirements of three standards and only one or two of them, respectively. The block diagram of the algorithm for creating an IMS in a design and survey organization is shown below in figure 1 .

As a tool for improving the efficiency of the IMS in the design and survey organization, a comprehensive approach is proposed based on the development of the business process system [5]. Improving the IMS should start with an analysis of each procedure in the system's processes to identify bottlenecks, shortcomings, and constraints that need to be addressed by 
performance improvement measures [6]. For each identified deviation that reduces the performance of the business process system, you must set a target value to which the system will strive to display indicators after implementing the planned improvement actions. Measures must be carried out sequentially from one constraint to another, since the effect of improving the IMS simultaneously for all deviations is equal to the increase in productivity only for one indicator that characterizes the operation of the system constraint, which, according to the Goldrat's Theory of Constraints, is unique at time t [7-9].

It is proposed to simplify the structure of the IMS of a design and survey organization in order to ensure traceability of operations and increase the efficiency of operation based on a comparison of the existing and target state of the system [10]. To adequately display the existing and target state of the IMS, we will build its mathematical model based on the identified limitations and system targets. To manage processes, it is necessary to develop a system of indicators that covers the performance and performance indicators of all processes identified in the system. Acceptable limits and target values of process performance indicators must necessarily correlate with each other within the system, with strategic indicators (goals) of the organization, customer requests (external and internal) [11]. Business process system management is based on the measurement of process performance indicators and their subsequent analysis. When analyzing business processes, their effectiveness, efficiency, and adaptability are determined. To form a General mathematical model for solving the problem of improving the efficiency of the design and survey organization, it is necessary to build three mathematical models: improving the efficiency, effectiveness and adaptability of the IMS functioning [12].

\section{Results}

The procedure for implementing an integrated approach to improving the efficiency of the IMS in the design and survey organization is shown below in figure 2.

1. Building a model for improving the efficiency of the IMS.

- target function: the efficient functioning of the IMS:

$$
\begin{aligned}
& \mathrm{Y}(\mathrm{n})=\left(\mathrm{Y}_{1}(\mathrm{n}), \mathrm{Y}_{2}(\mathrm{n}), \mathrm{Y}_{3}(\mathrm{n})\right) \rightarrow \max \\
& \quad\left(\mathrm{n}_{1}, \mathrm{n}_{2}, \mathrm{n}_{3}\right) \in \mathrm{Q}
\end{aligned}
$$

$\mathrm{n}_{1}$ - the criterion of effectiveness of the QMS;

$\mathrm{n}_{2}$ - the criterion of effectiveness EMS;

$\mathrm{n}_{3}$ - the criterion of effectiveness HaSMS;

$\mathrm{Q}$ - set of acceptable solutions for IMS performance. 
IMS analysis

-Analysis of the effectiveness of the business process of creating design and estimate documentation (DaED)

- Analysis of the effectiveness of the business process of creating DaED

- Analysis of financial and economic activity and its dependence on IMS

- Calculation of the increase in costs and profits from the IMS

\section{Improving the IMS}

- Identification of the effectiveness, efficiency and adaptability of the IMS functioning

- Formation of a set of measures to improve the efficiency, effectiveness and adaptability of the IMS functioning

Estimation of profit and cost increases

- Setting a link between audit results and weighting factors for profit and cost growth

- Calculating the increase in profit from the IMS

- Calculating the increase in IMS costs as a result of implementing measures

Search for solutions. Multi-criteria optimization problem statement:

- Determining the coefficient of profit growth from the operation of the IMS.

- Identification of limitations in the performance of the IMS.

- Determining the minimum required amount of costs for improving the IMS.

\section{Calculation of the expected economic effect}

- Calculation of the expected cumulative economic effect of the IMS

- Formation of measures to eliminate performance constraints

- Rational reallocation of costs for improving the IMS

Fig. 2. Structural and functional scheme of an integrated approach to improving the efficiency of the IMS in the design and survey organization

The minimum cost of creating design and survey products and the maximum economic effect is provided by focusing the business process system on the most effective crossfunctional interaction of the design and survey organization's divisions. Ensuring the development of cross-functional interaction of departments is carried out by highlighting and improving end-to-end processes. End - to-end process-a process that is implemented by several structural divisions of an organization. In the end-to-end process, the activities carried out are considered at the middle level, and the organization of control over the process and the results obtained is carried out by one manager. The effectiveness of a business process 
system depends on how manageable it is, i.e., on the amount of information available about its operation. Table 1 shows the key performance indicators of the IMS in the design and survey organization.

Table 1. Performance indicators of key IMS processes in a design and survey organization

Name of process
Development of design and estimate
documentation

Elimination of comments and remarks to the finished design and estimate documentation

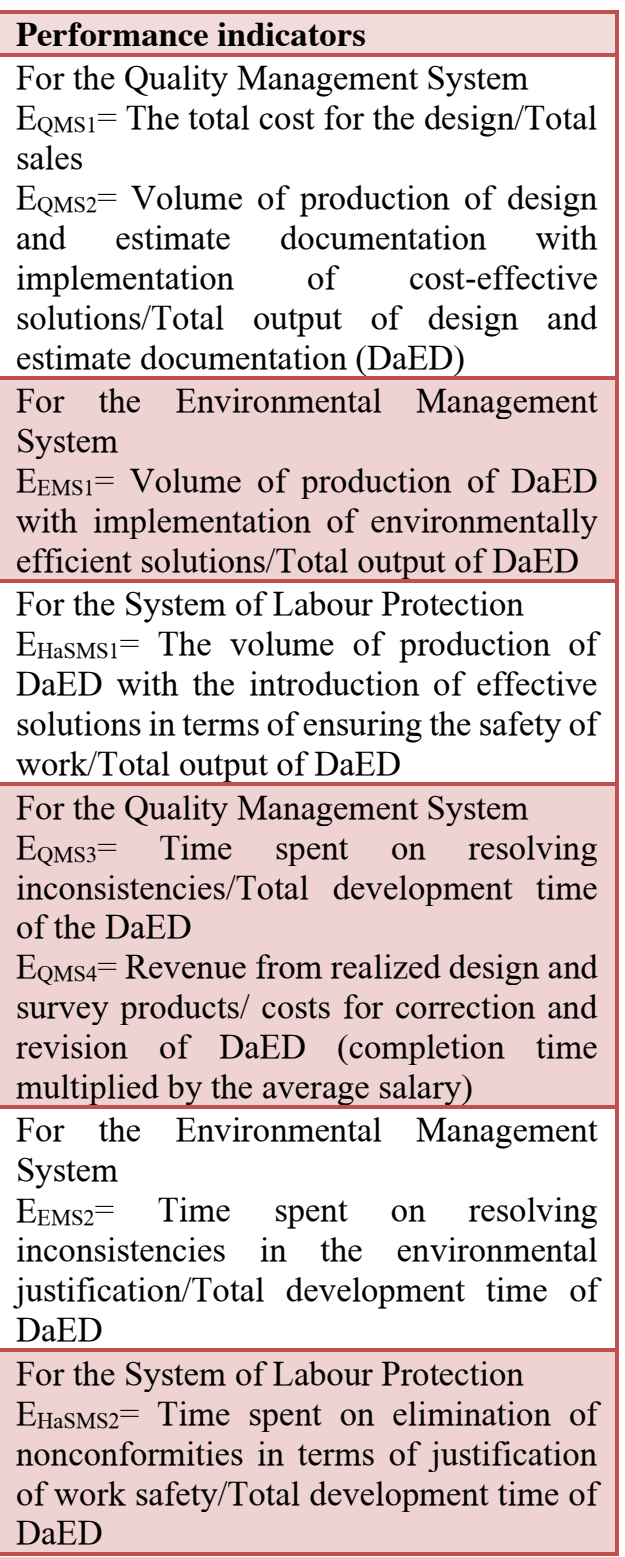

2. Building a model for improving the resultiveness of the IMS.

- target function: the efficient functioning of the IMS:

$$
\begin{aligned}
& \left.\mathrm{Z}(\mathrm{k})=\mathrm{Z}_{1}(\mathrm{k}), \mathrm{Z}_{2}(\mathrm{k}), \mathrm{Z}_{3}(\mathrm{k})\right) \rightarrow \max \\
& (\mathrm{k} 1, \mathrm{k} 2, \mathrm{k} 3) \in \mathrm{Q}
\end{aligned}
$$

Where $\mathrm{k}_{1}$ - the criterion of efficiency of the QMS; 
$\mathrm{k}_{2}$ - the criterion of efficiency of the EMS;

$\mathrm{k}_{3}-$ the criterion of efficiency of the HaSMS;

$\mathrm{Q}$ - set of acceptable solutions for IMS performance.

The effectiveness of the IMS, the actual results of operation of the processes set out in the plan is provided through continuous measurement of performance indicators of processes, analysis and implementing solutions for improvement. Performance evaluation allows you to determine the degree of compliance of business process outputs with customer requirements. The main indicators for assessing the effectiveness of management in a design and survey organization is the coefficient of implementation of the planned indicator of the economic effect of the project as a whole:

$$
\mathrm{K}_{\mathrm{e}}=\frac{\sum\left(C F_{i} \div(1+r)^{i}\right)}{\mathrm{E}}
$$

Where $\mathrm{E}$ - actual economic effect of the implemented project ; $\mathrm{CF}_{\mathrm{i}}-$ cost of free cash flow for period $\mathrm{i}$;

$r_{-}$discount rate;

i - sequential number of the discount period.

This figure characterizes the efficiency of design and survey organizations as a result of performance targets for the implementation of business process creation of project-estimate document, management, support processes, development processes.

3. Building a model for improving the adaptability of the ISM functioning.

- target function: the adaptiveness functioning of the IMS:

$$
\begin{aligned}
& \mathrm{G}(\mathrm{m})=(\mathrm{G} 1(\mathrm{~m}), \mathrm{G} 2(\mathrm{~m}), \mathrm{G} 3(\mathrm{~m})) \rightarrow \max \mid \\
& \left(\mathrm{m}_{1}, \mathrm{~m}_{2}, \mathrm{~m}_{3}\right) \in \mathrm{Q}
\end{aligned}
$$

Where $\mathrm{m}_{1}$ - the criterion of adaptibility of the QMS;

$\mathrm{m}_{2}$ - the criterion of adaptibility of the EMS;

$\mathrm{m}_{3}$ - the criterion of adaptibility of the HaSMS;

$\mathrm{Q}$ - set of acceptable solutions for IMS performance.

The business process adaptability assessment demonstrates how flexibly a given business process is able to respond to constantly changing environmental conditions and the needs of stakeholders. Flexibility in the proposed methodology is provided by implementing a component-portfolio approach that forms a rational structure of the design and survey organization's IMS. The formation of a portfolio of projects to improve the adaptability of the IMS is based on establishing an effective relationship between the processes by identifying three components of mobility: physical, financial, and functional (figure 3). 


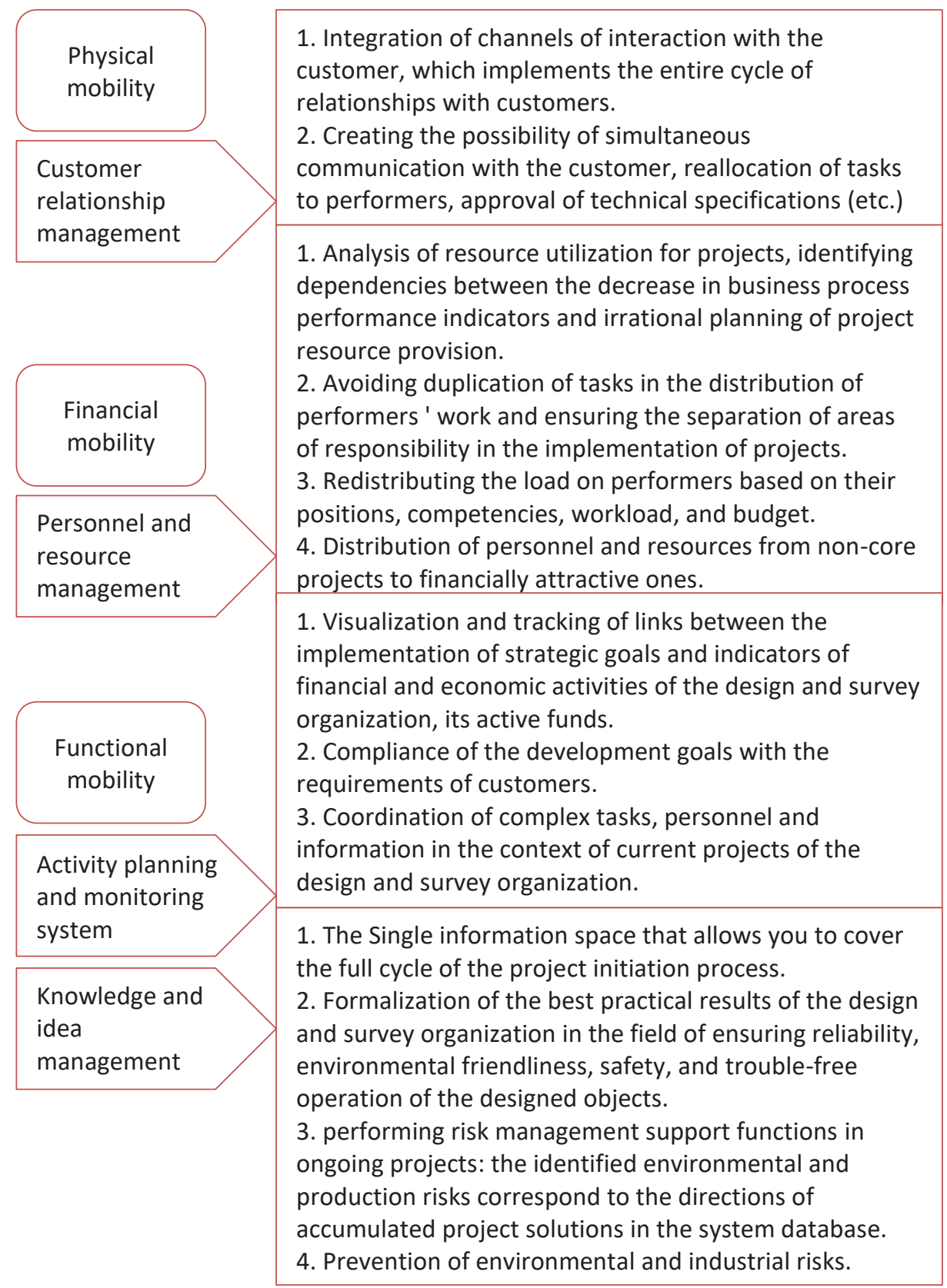

Fig. 3. Scheme for improving the adaptability of the IMS functioning

The method for solving the integrated management system improvement model is to set a multi-criteria optimization problem, which is focused on finding the optimal solution if there are three optimization criteria: efficiency, effectiveness and adaptability of the IMS functioning (figure 4): 


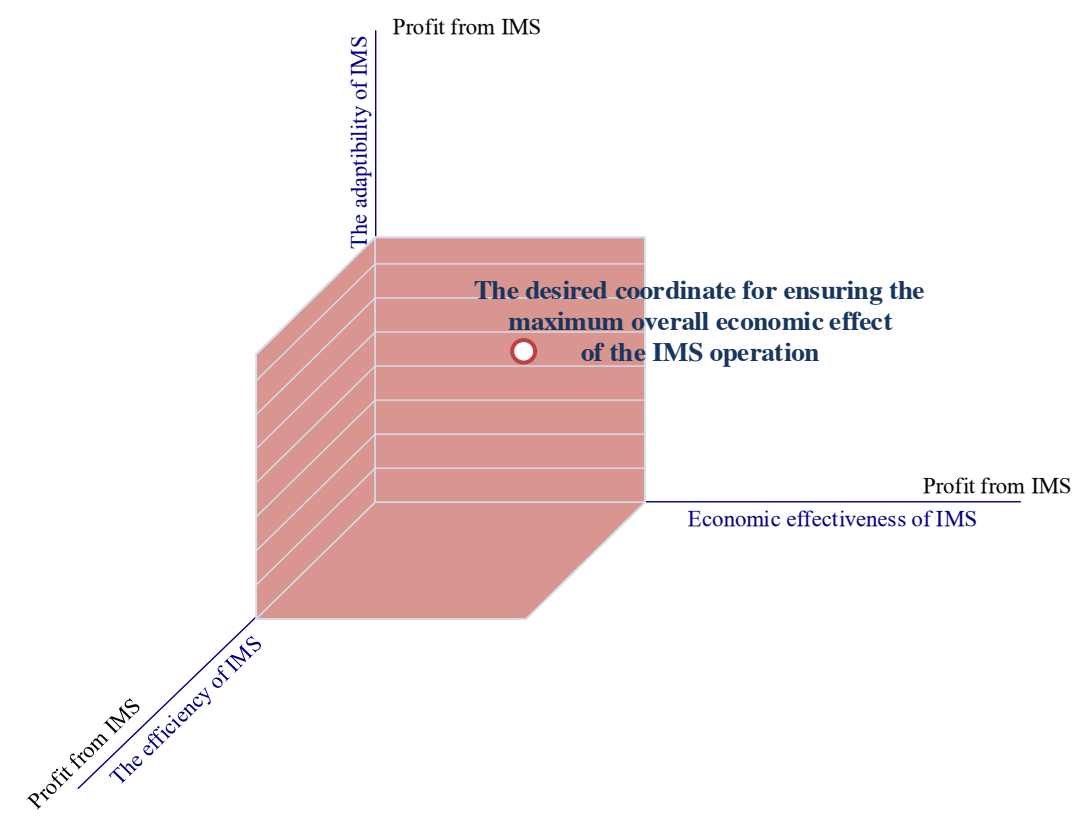

Fig. 4. Graphical representation of a model of an effectively functioning integrated management system, taking into account the desired coordinate

$$
\begin{aligned}
& \mathrm{F}(\mathrm{x})=\left(\mathrm{F}_{1}(\mathrm{x}), \mathrm{F}_{2}(\mathrm{x}), \mathrm{F}_{3}(\mathrm{x})\right) \rightarrow \max \\
& \left(\mathrm{x}_{1}, \mathrm{x}_{2}, \mathrm{x}_{3}\right) \in \mathrm{Q}
\end{aligned}
$$

$\mathrm{x}_{1}$ - the criterion of effectiveness of the IMS;

$\mathrm{x}_{2}-$ the criterion of efficiency of the IMS;

$\mathrm{x}_{3}$ - the criterion of adaptibility of the IMS;

$\mathrm{Q}$ - set of acceptable solutions for IMS performance.

Expert assessment of the increase in profits and costs as a result of the implementation of the developed measures is implemented in four stages:

1. Comparison of positive and negative audit results that characterize the functioning of the IMS and analysis results that characterize the effectiveness, efficiency and adaptability of the IMS.

2. establishing a link between the audit results and the corresponding weight coefficients of profit and cost growth.

3. analysis of the parameters of the design and survey organization and the developed set of measures to improve the functioning of the IMS.

4. calculation of the increase in profit and costs from the operation of the IMS as a result of the implementation of measures.

\section{Conclusion}

The results of the practical implementation of the developed methodology of effectiveness of the design and survey organization in terms of functioning of the showed an improvement in total economic impact from the operation of IMS through improved efficiency, 
effectiveness and adaptability of functioning of components of its quality management systems, environmental management and management of health and safety. The result of solving the mathematical models show that the maximum economic benefit depends on the limitations of the system not only for the costs of its implementation, analysis of which indicates a direct dependence of the growth of total costs for the development of an IMS and economic effect from implementing it.

\section{References}

1. W. E. Deming, The Essential Deming: Leadership Principles from the Father of Quality, McGraw-Hill Education (2012)

2. W. E. Deming, Some Theory of Sampling, Dover Publications (2010)

3. S. Thurner, R. Hanel, P. Klimek, Introduction to the Theory of Complex Systems, Oxford University Press (2018)

4. D. H. Meadows, Thinking in Systems, Chelsea Green Publishing (2008)

5. T. Boutros, The Basics of Process Improvement (New York, 2016)

6. F. Schönthaler, G. Vossen, A. Oberweis, T. Karle, Business Processes for Business Communities: Modeling Languages, Methods, Tools, Springer (2012)

7. E. Schragenheim, Management Dilemmas: The Theory of Constraints Approach to Problem Identification and Solutions, 2020 (1998)

8. W. H. Dettmer, Goldratt's Theory of Constraints: A Systems Approach to Continuous Improvement, North America (1997)

9. L. J. Scheinkopf, Thinking for a Change: Putting the TOC Thinking Processes to Use (1999)

10. W. H. Dettmer, Strategic Navigation: A Systems Approach to Business Strategy, North America (2003)

11. M. Armstrong, A. Baron, Performance Management, 2015, London, 11 (1998)

12. J. Ellenberg, How Not to Be Wrong: The Power of Mathematical Thinking, London. (2015) 